\title{
COMPARING BETWEEN RADIOTHERAPY PLANNING USING POSITRON EMISSION TOMOGRAPHY-COMPUTED TOMOGRAPHY (PET-CT) AND COMPUTED TOMOGRAFFYin (CT) IN HEAD AND NECK
}

\author{
A M Hussein ${ }^{(1)}$, O S Zehra ${ }^{(2)}$, H M Kohel ${ }^{(1)}$, A A Elsaid ${ }^{(2)}$ \\ (1) Department of Cancer Management and Research, Medical Research Institute. Alexandria University, Egypt, \\ (2) Department of Clinical Oncology and Nuclear Medicine, Faculty of Medicine, Alexandria University, Egypt,
}

\section{ABSTRA}

Objective: Conformal radiation therapy mandates accurate delineation of target volumes, which requires incorporation of modern imaging modalities like magnetic resonance imaging (MRI) and positron emission tomography (PET) in addition to conventionally used computed tomography (CT). This can resolve discrepancies in target delineation in head and neck carcinomas resulting in better local control.

Purpose: to compare between 18F-FDG-PET/CT versus CT scan in gross target volume volumes delineation in patients with Head and Neck cancer. Patients and Methods: 30 patients with pathologically confirmed primary HNSCC were enrolled. All patients had planning combined PET/CT in treatment position. All the GTVs (for the primary and nodal disease) were defined from both 18F FDG-PET/CT (GTV-PET) and CT (GTV-CT).

A visual interpretation method was used to delineate the margins of the GTV- PET.

The CTVs, as well as the PTVs, were created from the GTVs. All volumes had been measured and expressed in cubic centimeters.

Results: PET resulted in change of stage for 5 cases, 1 was up staged to metastatic disease was excluded from study treatment and was treated with palliative intent, but toxicity and response data is monitored, 2 was down staged.

GTVps delineated using CT scan (C-GTVp) were larger than those delineated using combined PET/CT scan (PET-GTVp) with median volume of $23.45 \mathrm{cc}$ compared to $16.67 \mathrm{cc}$ of (C-GTV) and (PET-GTV) respectively however didn't reach the statistical significance value $(\mathrm{P}=$ $0.074)$.

GTVns delineated using CT scan (C-GTVn) were smaller than those delineated using combined PET/CT scan (PET-GTVn) with median volume of $6.85 \mathrm{cc}$ compared to $10.67 \mathrm{cc}$ of (C-GTVn) and (PET-GTVn) respectively with statistical significance $(\mathrm{P}=0.045)$.

Conclusion: the use of PET/CT resulted in change of disease's stage, the primary tumor and nodal GTVns were changed with the implementation of combined PET/CT; that translate into a smaller and statistically significant difference in GTVns of the nodal disease, however, such difference in the volumes was not statistically significant when applied to primary tumor GTVs, CTVs and PTVs..

Keywords: (18F-FDG-PET/CT scanning, Head and Neck cancer, GTV-PET, GTV-CT, target volumes delineation, gross tumor volume).

\section{INTRODUCTION}

Head and neck cancers (HNCs) are the sixth most common cancers worldwide causing 300,000 deaths annually, it accounts for $3 \%$ of all cancers in the developed countries like USA and $30 \%$ of all cancers in developing countries such as Egypt. (1)

Head and neck squamous cell carcinoma (HNSCC) comprise the vast majority of head and neck cancer (HNC), radiation therapy along with eoneurrent ehemotherapy is the standard treatment for most of the locally advanced head and neck squamous cell carcinoma. Computed tomography (CT) is the conventionally used imaging modality to delineate the tumor for 3-D treatment planning as it provides information about the tissue densities in the form of electron density which is required for radiotherapy dose calculation. ${ }^{(2)}$

The incorporation of PET in radiotherapy treatment planning has revolutionized the field of radiation oncology. With the introduction of advanced radiotherapy treatment techniques like 3-D conformal radiotherapy (3-D CRT) and intensity modulated radiotherapy (IMRT), it is of utmost importance to delineate the target volume precisely in order to achieve a good tumor control. To utilize PET in radiotherapy, integrated PET-CT is the best option, it provides precise localization of lesions and improves the standard ization of volume delineation compared with that of CT alone ${ }^{.(3)}$

Using PET-CT in radiotherapy planning reducing inter observer variability in target delineation, and modifying the extension of tumor volumes including gross (GTV), clinical (CTV) and planning target volumes (PTV) and consequently allowing additional dose escalation for both primary tumor and regional lymph nodes. ${ }^{(1-4)}$

The aim of this study is to compare between combined 18F-FDG-PET/CT versus CT scan in gross target volumes delineation, in patients with Head and Neck Squamous cell carcinoma treated by radiotherapy with or without chemotherapy. 


\section{PATIENTS AND METHODS}

\section{1- Patients Eligibility}

Between April 2017 and March 2018, 30 patients with primary pathologically confirmed squamous cell carcinoma of the head and neck were prospectively enrolled in the study, their ages ranged between 19 and 71 years old, all had measurable disease of stages I, II, III, IVa, classified according to the International Union Against Cancer staging criteria (8th edition 2017).

Performance status according to ECOG scale was $\leq 1$ and all patients provided an informed consent for treatment, the study was approved by the Ethical Committee of Scientific Research- Faculty of Medicine- Alexandria University.

All patients had pretreatment evaluation including: Complete medical history, Complete physical examination, assessment of the performance status according to ECOG scales. Biopsies from the primary lesion and lymph nodes were performed for histopathological confirmation. $\mathrm{CBC}$, renal functions, liver function, and creatinine clearance were evaluated. All patients underwent diagnostic C.T scan and/or MRI of primary site and regional lymph nodal areas. Pretreatment dental evaluation and prophylaxis were done.

\section{2-18F-FDG-PET/CT Image Acquisition}

All the patients were immobilized with a customized thermoplastic mask fixed to a flat table, PET/CT was done using a standard procedure and each patient had fasted for $\geq 6 \mathrm{~h}$ and was injected with18F-FDG, images were obtained 60-80 min after injection. The scan was performed with a dedicated PET-CT scanner.

The field of view of PET/CT was from the skull to the thigh, PET/CT acquisition was performed in the treatment position, the data sets obtained and imported on a workstation treatment planning system.

\section{Delineation of Target Volumes}

According to ICRU 62 report, Physical examination, Contrast-enhanced CT and/or MRI, of the primary tumor and regional disease had been used to tailor the radiotherapy portals and target volumes, GTV: gross tumor volume (primary or nodal disease), CTV (clinical target volume): including GTV + margin (according to the site), potential routes of spread and the entire lymphatic drainage of the neck, PTV (the planning target volume): is CTV $+5 \mathrm{~mm}$ margin.
All the GTVs (for the primary and nodal disease) were defined from both 18F-FDG-PET/CT (GTV- PET) and CT (GTVCT). The CT-based GTV was drawn without the information from the FDG-PET scans. The 18F-FDGPET-positive regions were interpreted as malignant on the emission images. A visual method used to delineate the margins of the GTV PET. The CTVs, as well as the PTVs, were created from the GTVs. All volumes had been measured and expressed in cubic centimeters.

\section{.4. Response evaluation}

was evaluated according to Response Evaluation Criteria in Solid Tumors (RECIST) version 1.1

\section{.5 Statistical methods}

Data were analyzed using SPSS 17 program with paired (t) test was used to asses Significant of difference between pairs of measures.

\section{THE OBJECTIVE}

was to analyze the differences in tumor volumes obtained by the two modalities of measurement during conformal radiotherapy planning, CT and combined 18F-FDG$\mathrm{PET} / \mathrm{CT}$.

\section{RESULTS}

30 patients, 23 men $(76.7 \%)$ and 7 women $(23.3 \%)$ as showed in table 1 were prospectively enrolled in the study between April 2017 and March 2018. The age ranged between 25-70 year, the median age was 54.5 year and a mean age of $53.13 \pm 10.41$ as shown in table 2 .

Table (1): Distribution of patients according to sex

\begin{tabular}{lll}
\hline \multicolumn{1}{c}{ Sex } & Frequency & Percentage \\
\hline Male & 22 & $\mathbf{7 3 . 3}$ \\
Female & 8 & $\mathbf{2 6 . 6}$ \\
Total & 30 & $\mathbf{1 0 0}$ \\
\hline
\end{tabular}

Regarding the primary site, 11 patients (36.6\%) had nasopharyngeal carcinoma, 6 patients $(20 \%)$ with cancer of the oropharynx. $5(16.6 \%)$ patients had oral cavity tumors, 5 patients $(16.6 \%)$ had laryngeal cancer, another 2 patients $(6.6 \%)$ presented with carcinoma of the hypopharynx while only one patient had cervical lymphadenopathy of unknown primary represents $3 \%$ of the cases as shown in figure 1.

Table (2): Age of patients upon recruiting in the study .

\begin{tabular}{lllll}
\hline Age (years) & Frequency & Percentage & Cumulative Percentage \\
\hline $18-30$ yrs. & 3 & 10 & $\mathbf{1 0}$ \\
$31-50$ yrs. & 12 & 40 & $\mathbf{5 0}$ \\
$51-70$ yrs. & 14 & & 46.66 & $\mathbf{9 6 . 6 6}$ \\
& $\geq 71$ yrs. & 1 & 3.33 & $\mathbf{1 0 0}$ \\
& Total & 30 & 100 & \\
\hline
\end{tabular}




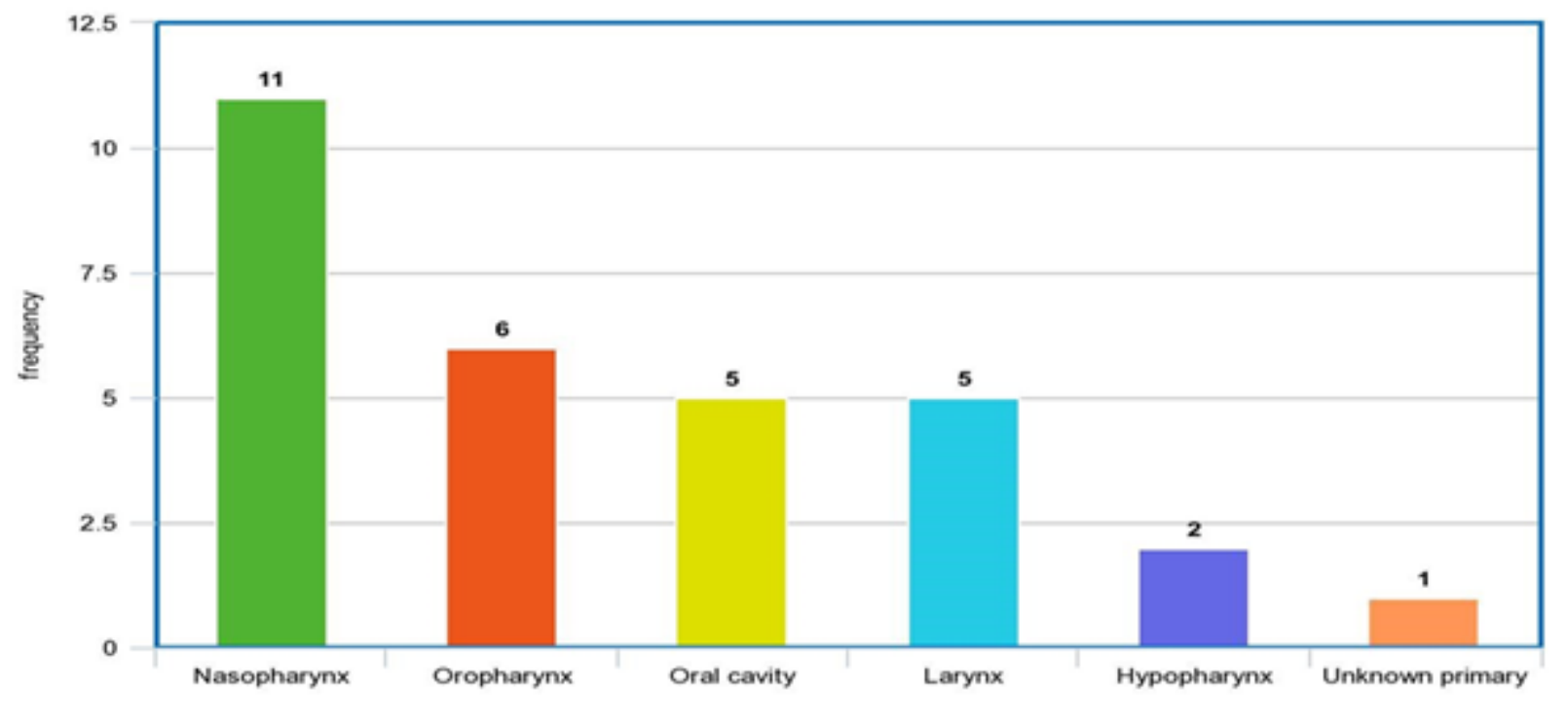

Figure (1): Distribution of patients according to primary tumor site in head and neck region.

Table (3) shows that GTVs delineated using CT scan (CT-GTVp) were larger than those delineated using combined PET/CT scan (PET-CT GTVp) with median volume of $32.1 \mathrm{cc}$ compared to $18.5 \mathrm{cc}$ of (CT-GTVp) and (PET-CT GTVp) respectively with high statistical significance $(\mathrm{P}=0.0026)$. (PET-GTV) was smaller than (C-GTV) by a mean of $10.01 \mathrm{cc}$ which represents $30.08 \%$ reduction in the gross tumor volume using PET/CT compared to clinical and traditional imaging modalities. The clinical target volumes delineated using CT scan (CT based CTV) and PET/CT scan (PET-CTV) were compared, the PET-CTV was smaller than CT based CTV but yet non-statistically significant $(\mathrm{P}=0.58)$, shown in table 4 .

The PTVs drown using CT scan were comparable to PTVs delineated using PET/CT scan $(\mathrm{P}=0.51)$, table 5.

Table (3): analysis of the study cases according to PET-CT GTVp/ CT GTVp (n):30

\begin{tabular}{|c|c|c|c|c|}
\hline & CT GTVp & PET-CT GTVp & $t$ value & $P$ value \\
\hline Min-max & $3.5-51.4$ & $2.1-46.5$ & & \\
\hline Mean \pm SD & $30.9 \pm 13.68$ & $20.89 \pm 10.54$ & 3.15 & $0.0026^{*}$ \\
\hline Median & $32.1 \mathrm{cc}$ & $18.5 \mathrm{cc}$ & & \\
\hline change & \multicolumn{2}{|c|}{ Decrease 10.01cc } & & \\
\hline$\%$ of change & \multicolumn{2}{|c|}{$30.08 \%$} & & \\
\hline
\end{tabular}

*p value of paired t test comparing between PET-CT GTVp/ CT GTVp.

Table (4): analysis of the study cases according to PET-CT CTV/ CT CTV,(n):30

\begin{tabular}{lllll}
\hline & CT CTV & PET-CT CTV & t value & P value \\
\hline Min-max & $160.5-540.3 \mathrm{cc}$ & $\mathbf{1 5 5 . 3 - 5 3 8}$ & & \\
Mean \pm SD & $329.67 \pm 70.3$ & $\mathbf{3 2 6 . 8 4} \pm 69.8$ & $\mathbf{t}=\mathbf{0 . 2 1 8}$ & $0.58^{*}$ \\
Median & $312 \mathrm{cc}$ & $\mathbf{3 0 2 \mathrm { cr }}$ & \\
\hline
\end{tabular}

*P value of paired t test comparing between PET-CT CTV/ CT CTV. 
Table (5): analysis of the study cases according to PET-CT PTV/ CT PTV, (n):30

\begin{tabular}{llll}
\hline & CT PTV & PET-CT PTV & P value \\
\hline Min-max & $312.2-914.33 \mathrm{cc}$ & $\mathbf{3 1 1 . 4 - 9 1 2 . 2 c c}$ & $\mathbf{t = 0 . 0 3 9}$ \\
Mean \pm SD & $618.2 \pm 139.2$ & $\mathbf{6 1 7 . 2} \pm \mathbf{1 3 8 . 9}$ & $\mathbf{0 . 5 1 *}$ \\
Median & $634.1 \mathrm{cc}$ & $\mathbf{6 3 3 . 1 \mathrm { cc }}$ & \\
\hline
\end{tabular}

${ }^{*} \mathrm{P}$ value of paired $\mathrm{t}$ test comparing between PET-CT PTV/ CT PTV.

\section{DISCUSSION}

The incorporation of PET in radiotherapy treatment planning has revolutionized the field of radiation oncology, the therapeutic index in radiation therapy can be improved with better target volume delineation. ${ }^{(1)}$

This is especially true in head and neck cancers where organs at risk are in close proximity to these targets and highly conformal therapies are often needed to minimize morbidity.

In recent years, more research has been made in evaluating the clinical role of combined ${ }^{18} \mathrm{~F}$ FDG PET/CT on target volume delineation in head and neck cancer. ${ }^{(2-4)}$ Although some groups report that ${ }^{18}$ F-FDG-PET/CT based delineation resulted in smaller tumor volumes than the CT-based volumes, others report that GTV defined with ${ }^{18}$ F-FDG PET/CT was similar or even larger than their CT-based counterparts. ${ }^{(5-9)}$

The present study was performed to compare between ${ }^{18}$ F-FDG-PET/CT versus CT scan in target volumes delineation, in patients with head and neck cancer treated by radiotherapy with or without chemotherapy. Thirty patients with pathologically proven head and neck cancer were included in this prospective study which was conducted between April 2017 and March 2018.

in our study, fused ${ }^{18} \mathrm{~F}$-FDG-PET/CT images produce different target volumes to those produced using conventional method CT, it was found that GTVs delineated using CT scan (C-GTV) were larger than those delineated using PET/CT (PET-GTV) scan in most of the cases $(83.3 \%)$, while in 5 cases $(6.67 \%)$ only the PETGTV was greater than C-GTV unlike the results of Burela's study in which the GTV-PET was larger than GTV-CT in 15 cases $(55.56 \%)$ and was smaller in 12 cases $(44.44 \%){ }^{(10)}$

In our study, the median volume of C-GTV was 32.1cc and $18.5 \mathrm{cc}$ of PET-GTV compared to a median value of $11.31 \mathrm{cc}$ for GTV-CT in Burela's, while that of GTV-PET was $13.81 \mathrm{cc}$. GTV delineated on PET vs. CT scan for primary tumor and nodes showed no statistically significant change in the mean volumes $(P=0.803$ for GTV primary, $\mathrm{P}=0.589$ for nodes $).{ }^{(10)}$
In our study, the volume difference between GTVs using CT scan versus PET/CT, was highly statistically significant for the primary tumor $((\mathrm{P}=<0.001)$. but not significant for the nodal volumes $(\mathrm{P}=0.171$ for neck nodes).

Delouya et al. 2011, studied twenty-nine patients with SCC of the head and neck to determine the impact of ${ }^{18} \mathrm{~F}$ FDGPET in radiotherapy target delineation and patient management, compared to CT alone, all gross tumor volumes of the primary (GTVp) and the suspicious lymph nodes (GTVn) were defined on CT (GTVp-CT) and ${ }^{18} \mathrm{~F}$ FDG-PET/CT (GTVp-PET),four patients who had previous diagnostic tonsillectomies were excluded, for the other 25 patients, GTVp-PET were smaller than the GTVp-CT in $80 \%$ of the cases, leading to a statistically significant volume difference $(\mathrm{p}=0.001)$.no such volume change was observed $(\mathrm{p}=0.08)$ for the lymph node, ${ }^{18} \mathrm{~F}$ FDG-PET modified treatment management in three patients (Distant metastases were found in two patients and one had a newly diagnosed lung adenocarcinoma), these results are compatible with the current study. ${ }^{(11)}$

Similarly, Guido et al. 2009, found that in $92 \%$ of cases, the CT-based GTVs were larger than the PET/CT-based GTVs.The average total GTV from the CT and PET/CT scans was $34.54 \mathrm{~cm} 3$ (range, 3.56-109) and $29.38 \mathrm{~cm} 3$ (range, 2.87-95.02), respectively ( $\mathrm{p}<0.05)$. Separate analyses of the difference between the CT- and PET/CTbased GTVs of nodal disease were not statistically significant. $^{(5)}$

Paulino et al. (2005), investigated 40 patients and found changes in the GTV in 37 of 40 cases. Of the 40 patients, 30 had a decrease in GTV on PET/CT and 7 had an increase. However, in that study, the investigators fused both the CT portion of the PET-CT and the CT simulation images, thus introducing a possible source of error, furthermore, a separate analysis of the data according to primary tumor and nodal disease was not provided. ${ }^{(6)}$

Heron et al. (2004), enrolled 21 patients with head-andneck cancer. Overall, $80 \%$ of all patients presented with a change in the GTV delineation. The investigators performed a separate analysis of the primary tumor vs. nodal disease and found that in the primary tumor, the GTV was increased in 3 of 21 patients and decreased in 
14, in the nodal stations, it was increased in 7 and decreased in 3 of 21 patients. ${ }^{(7)}$

\section{Conclusion \& Recommendations}

Combined PET/CT imaging-utilizing anatomical and biological data- could improve target volume delineation and RT dosimetry and, likely, provide better locoregional control in head-and neck cancer while sparing the surrounding normal tissues.

In our study, the primary tumor and nodal GTVs were changed with the implementation of combined PET/CT; that translate into a smaller and statistically significant difference in the mean volume of the GTV of the primary tumor, however such difference in the volumes was not statistically significant when applied to nodal GTVs, CTVs and PTVs.

Larger multicenter studies are needed to ascertain whether combined 18F-FDG-PET/CT in target delineation can influence the main clinical outcomes, such as toxicity reduction, dose escalation to smaller volumes without compromising local control, and survival.

We recommend to perform combined PET/CT in the GTV delineation of all head-and neck cancer patients as implementation of combined PET/CT imaging has the potential to improve primary tumor and nodal basins, target delineation and clarify uncertain lesions.

The role of novel PET tracers, designed to depict biological characteristics such as hypoxia (18-F fluoromisonidazole $=$ FMISO), protein synthesis $(11 \mathrm{C}$ methionine, MET), and cell proliferation (32-deoxy-32(18) F-fluoro- thymidine, FLT) in HNC patient care, seems to be a good field for future researches.

One or more of these tracers may one day facilitate functional imaging-based individualization of treatment strategies

\section{REFERENCES}

1. Al-Ibraheem, A, Buck, A, Joachim Krause B, Scheidhauer K. Clinical Applications of FDG PET and PET/CT in Head and Neck Cancer. Journal of Oncology, 2009.
2. Bujenovic S.The role of positron emission tomography in radiation treatment planning. Seminars in Nuclear Med 2004 34, 293-9.

3. Solberg T, Agazaryan N, GossW.A feasibility study of 18F-fluorodeoxyglucose positron emission tomography targeting and simultaneous integrated boost for intensity-modulated radiosurgery and radiotherapy. Journal of Neurosurgery 2004, 101, 381-9.

4. .Czernin J,Phelps T.Positron emission tomography scanning: Current and future applications. Annual Review of Medicine 2002, 53:89-112.

5. .Guido A, Fuccio L,Rombi B.Combined 18F-FDGPET/CT imaging in radiotherapy target delineation for head and neck cancer. International Journal of Radiation Oncology, Biology, Physics 2005, 73, 759-63.

6. .Paulino A.C, Koshy M, Howell R. Comparison of CTand FDG-PET-defined gross tumor volume in intensitymodulated radiotherapy for head-and-neck cancer. International Journal of Radiation Oncology, Biology, Physics 2005, 61, 1385-92.

7. .Heron D. E, Andrade R. S, Flickinger J. Hybrid PETCT simulation for radiation treatment planning in headand-neck cancers: A brief technical report. International Journal of Radiation Oncology, Biology, Physics 2004, 60, 1419-24.

8. Chauhan D, Rawat S, Sharma M. K, Ahlawat P, Pal M, Gupta, G. Improving the accuracy of target volume delineation by combined use of computed tomography, magnetic resonance imaging and positron emission tomography in head and neck carcinomas. Journal of Cancer Research and Therapeutics 2005, 11, 746-51.

9. Deantonio L, Beldì D, Gambaro G, Loi G, Brambilla M. FDG-PET/CT imaging for staging and radiotherapy treatment planning of head and neck carcinoma. Radiation Oncology Journal 2008 , 3, 29-34.

10. Burela N, Soni T.P, Patni N, Bhagat J.K,Kumar T.S. A quantitative comparison of gross tumor volumes delineated on[18F]-FDG-PET/CT scan and contrastenhanced computed tomography scan in locally advanced head and neck carcinoma treated with Intensity Modulated Radiotherapy. Advances in Modern Oncology Research 2017, 3(3), 143-51.

11. Delouya G, Igidbashian L, Houle A, Bélair M, Boucher $\mathrm{L}$, Cohade $\mathrm{C}$, et al. ${ }^{18} \mathrm{~F}-\mathrm{FDG}-\mathrm{PET}$ imaging in radiotherapy tumor volume delineation in treatment of head and neck cancer. Radiotherapy and Oncology 2016,11,211-9 